\title{
Deep Xmm-newton Spectroscopic and Timing Observations of the Isolated Radio Millisecond Pulsar Psr J0030+0451
}

\section{Citation}

Bogdanov, Slavko, and Jonathan E. Grindlay. 2009. "DEEP XMM-NEWTON SPECTROSCOPIC AND TIMING OBSERVATIONS OF THE ISOLATED RADIO MILLISECOND PULSAR PSR J0030+0451."

The Astrophysical Journal 703 (2): 1557-64. https://doi.org/10.1088/0004-637x/703/2/1557.

\section{Permanent link}

http://nrs.harvard.edu/urn-3:HUL.InstRepos:41399742

\section{Terms of Use}

This article was downloaded from Harvard University's DASH repository, and is made available under the terms and conditions applicable to Open Access Policy Articles, as set forth at http:// nrs.harvard.edu/urn-3:HUL.InstRepos:dash.current.terms-of-use\#OAP

\section{Share Your Story}

The Harvard community has made this article openly available. Please share how this access benefits you. Submit a story.

Accessibility 
Draft version November 20, 2018

Preprint typeset using $\mathrm{L}_{\mathrm{T}}^{\mathrm{E}} \mathrm{X}$ style emulateapj v. 08/22/09

\title{
DEEP XMM-NEWTON SPECTROSCOPIC AND TIMING OBSERVATIONS OF THE ISOLATED RADIO MILLISECOND PULSAR PSR J0030+0451
}

\author{
Slavko Bogdanov ${ }^{1,2,3}$ And Jonathan E. GRINDlay ${ }^{1}$ \\ Draft version November 20, 2018
}

\begin{abstract}
We present deep XMM-Newton EPIC spectroscopic and timing X-ray observations of the nearby solitary radio millisecond pulsar, PSR J0030+0451. Its emission spectrum in the $0.1-10 \mathrm{keV}$ range is found to be remarkably similar to that of the nearest and best studied millisecond pulsar, PSR J0437-4715, being well described by a predominantly thermal two-temperature model plus a faint hard tail evident above $\sim 2 \mathrm{keV}$. The pulsed emission in the $0.3-2 \mathrm{keV}$ band is characterized by two broad pulses with pulsed fraction $\sim 60-70 \%$, consistent with a mostly thermal origin of the X-rays only if the surface polar cap radiation is from a light-element atmosphere. Modeling of the thermal pulses permits us to place constraints on the neutron star radius of $R>10.7$ (95\% confidence) and $R>10.4$ $\mathrm{km}$ (at $99.9 \%$ confidence) for $M=1.4 \mathrm{M}_{\odot}$.
\end{abstract}

Subject headings: pulsars: general — pulsars: individual (PSR J0030+0451) — stars: neutron — X-rays: stars - relativity

\section{INTRODUCTION}

PSR J0030+0451 is one of the nearest known rotationpowered "recycled" millisecond pulsars (MSPs) in the field of the Galaxy $(D=300 \pm 90 \mathrm{pc}$; Lommen et al. 2006), with a spin period $P=4.87 \mathrm{~ms}$ and intrinsic spindown rate $\dot{P} \equiv \mathrm{d} P / \mathrm{d} t=1.0 \times 10^{-20} \mathrm{~s} \mathrm{~s}^{-1}$, implying a surface dipole magnetic field strength $B \approx 2.7 \times 10^{8}$ Gauss, a characteristic age $\tau \approx 7.8 \mathrm{Gyr}$, and spin-down luminosity $\dot{E} \approx 3 \times 10^{33} \mathrm{ergs} \mathrm{s}^{-1}$. This solitary MSP was discovered at radio frequencies in the Arecibo drift scan survey (Lommen et al. 2000). Recently, a firm detection of PSR J0030+0451 at $\gamma$-ray energies by the Fermi Large Area Telescope was reported by Abdo et al. (2009). In X-rays, PSR J0030+0451 has been detected with ROSAT PSPC (Becker et al. 2000) and XMMNewton (Becker \& Aschenbach 2002). Despite the limited photon statistics, these observations clearly showed two distinct broad pulses. In addition, the XMM-Newton observation revealed a relatively soft $0.3-2 \mathrm{keV}$ spectrum, qualitatively similar to that of PSR J0437-4715 (Zavlin 2006).

X-ray observations have detected a number of rotationpowered MSPs that exhibit soft, presumably thermal, emission (Zavlin 2006; Bogdanov et al. 2006a; Zavlin 2007). This radiation likely originates from the pulsar magnetic polar caps that are heated by energetic particles from the pulsar magnetosphere (see, e.g., Harding \& Muslimov 2002). As this heat is confined to a small portion of the NS, study of the X-ray properties of MSPs could offer insight into key NS properties that are inaccessible by other observational means (e.g., radio pulse timing) such as the radiative properties of the NS surface, magnetic field geometry, and NS compactness $\left(R / R_{S}\right.$, where $R_{S}=2 G M / c^{2}$ and $R$

\footnotetext{
${ }^{1}$ Harvard-Smithsonian Center for Astrophysics, 60 Garden Street, Cambridge, MA 02138; sbogdanov@cfa.harvard.edu, josh@cfa.harvard.edu

2 Department of Physics, McGill University, 3600 University Street, Montreal, QC H3A 2T8, Canada

${ }^{3}$ Canadian Institute for Advanced Research Junior Fellow
}

and $M$ are the stellar mass and radius). As shown by Pavlov \& Zavlin (1997), Zavlin \& Pavlov (1998), and Bogdanov et al. (2007), a model of polar cap thermal emission from an optically-thick hydrogen (H) atmosphere provides a good description of the X-ray pulse profiles of PSR J0437-4715, the nearest known MSP. On the other hand, a blackbody model is inconsistent with the pulsed X-ray emission. Furthermore, there is compelling evidence for a magnetic dipole axis offset from the NS center (Bogdanov et al. 2007). Finally, the compactness of PSR J0437-4715 is constrained to be $R / R_{S}>1.6$ (99.9\% confidence), which for the current best mass measurement $\left(1.76 \mathrm{M}_{\odot}\right.$, see Verbeist et al. 2008) implies $R>8.3 \mathrm{~km}$. Bogdanov et al. (2008) have shown, using short archival XMM-Newton observations of PSRs J0030+0451 and J2124-3358, that even with relatively crude photon statistics, it is possible to set limits on $M / R$. In particular, assuming $1.4 \mathrm{M}_{\odot}$, the stellar radius is constrained to be $R>9.4 \mathrm{~km}$ and $R>7.8 \mathrm{~km}$ (68\% confidence) for each pulsar, respectively. Thus, realistic modeling of X-ray data from MSPs appears to be a promising approach towards revealing the structure of NSs and warrants further X-ray observations of nearby MSPs. As one of the nearest known recycled pulsars, PSR J0030+0451 is well suited for such an investigation.

In this paper, we present an analysis of deep XMMNewton spectroscopic and timing observations of PSR J0030+0451. These observations provide new insight into the X-ray properties of MSPs and the structure of neutron stars. The work is presented as follows. In $\S 2$ we summarize the observations and data reduction procedure. In $\S 3$ we briefly examine the X-ray images. In $\S 4$ we constrain the spectral properties of the pulsar, while in $\S 5$ we conduct a timing analysis. In $\S 6$ we attempt to model the pulsed emission from the MSP. We offer conclusions in $\S 7$.

\section{OBSERVATION AND DATA REDUCTION}

PSR J0030+0451 was targeted by XMM-Newton between 12 and 14 December 2007 (observation ID 050229). 


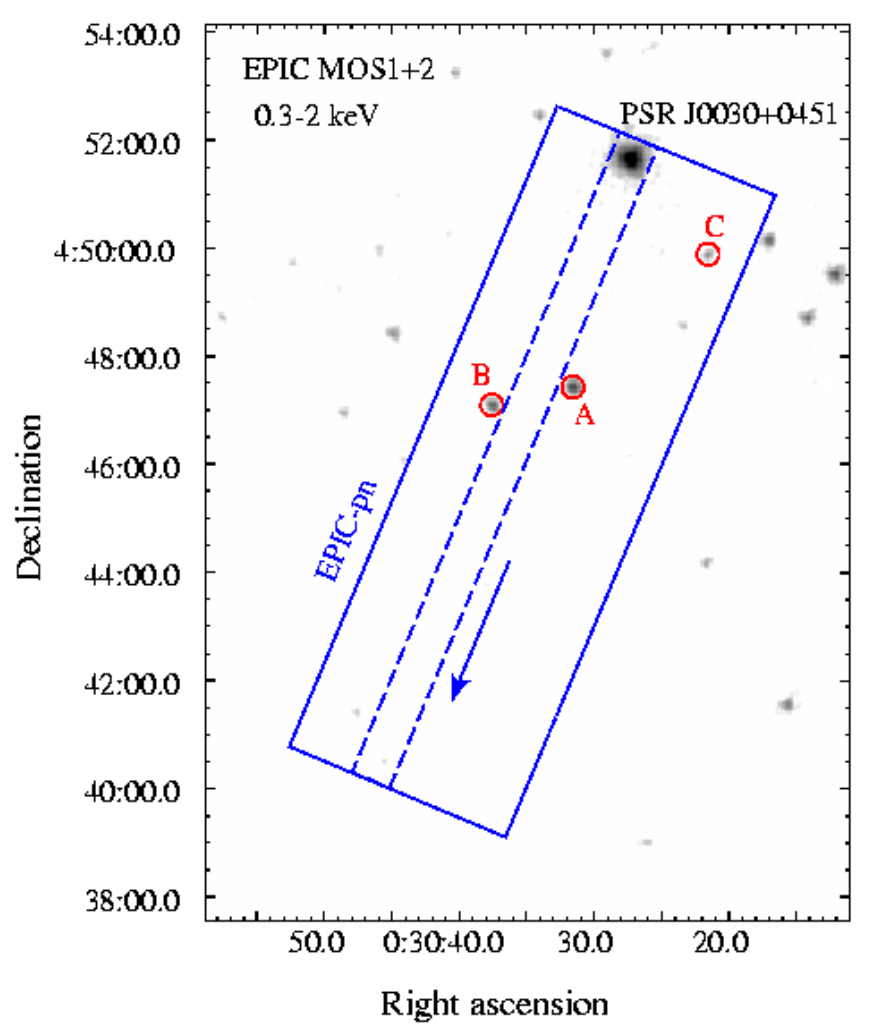

FIG. 1.- XMM-Newton MOS1 and MOS2 mosaic image of the field near PSR J0030+0451 in the $0.3-2 \mathrm{keV}$ band. The rectangle shows the relative position and orientation of the active EPIC pn chip used in this observation, with the timing redout direction indicated by the arrow. The dashed lines correspond to the EPIC pn detector columns used to extract the source counts from the pulsar while the circles mark the positions of sources that fall within the EPIC pn field of view.

The observation was carried out in a single uninterrupted 130-kilosecond exposure, corresponding to the entire usable observing time of XMM-Newton revolution 1467. The European Photon Imaging Camera (EPIC) MOS1 and MOS2 instruments were configured for full imaging mode. The EPIC pn was configured for fast timing mode, in which only CCD 4 is active, allowing $30 \mu$ s time resolution at the expense of one imaging dimension. For all three instruments, the thin optical filter was used. Due to the faint nature of J0030+0451, the dispersed Reflection Grating Spectrometer (RGS) data provide no useful spectral or timing information. Therefore, the grating data were not used in our analysis.

The data reduction, imaging, and timing analyses were performed using the $\mathrm{SAS}^{4}$ 8.0.1 and FTOOLS 5 6.6.1 software packages, while the spectral analysis was conducted in $\mathrm{XSPEC}^{6}$ 12.5.0. The raw MOS and pn datasets were first reprocessed using the SAS emchain and epchain pipelines, respectively, and subsequently screened for instances of high background proton flares. A total of $\sim 36$ ks of high-background data were discarded, resulting in 92.5, 92.1, and $92.1 \mathrm{ks}$ of usable exposure time for MOS1, MOS2, and pn, respectively. Finally, the appropriate

\footnotetext{
4 The $X M M$-Newton SAS is developed and maintained by the Science Operations Centre at the European Space Astronomy Centre and the Survey Science Centre at the University of Leicester.

5 Available at http://heasarc.gsfc.nasa.gov/ftools/

6 Available at http://heasarc.nasa.gov/docs/xanadu/xspec/
}

ndlay

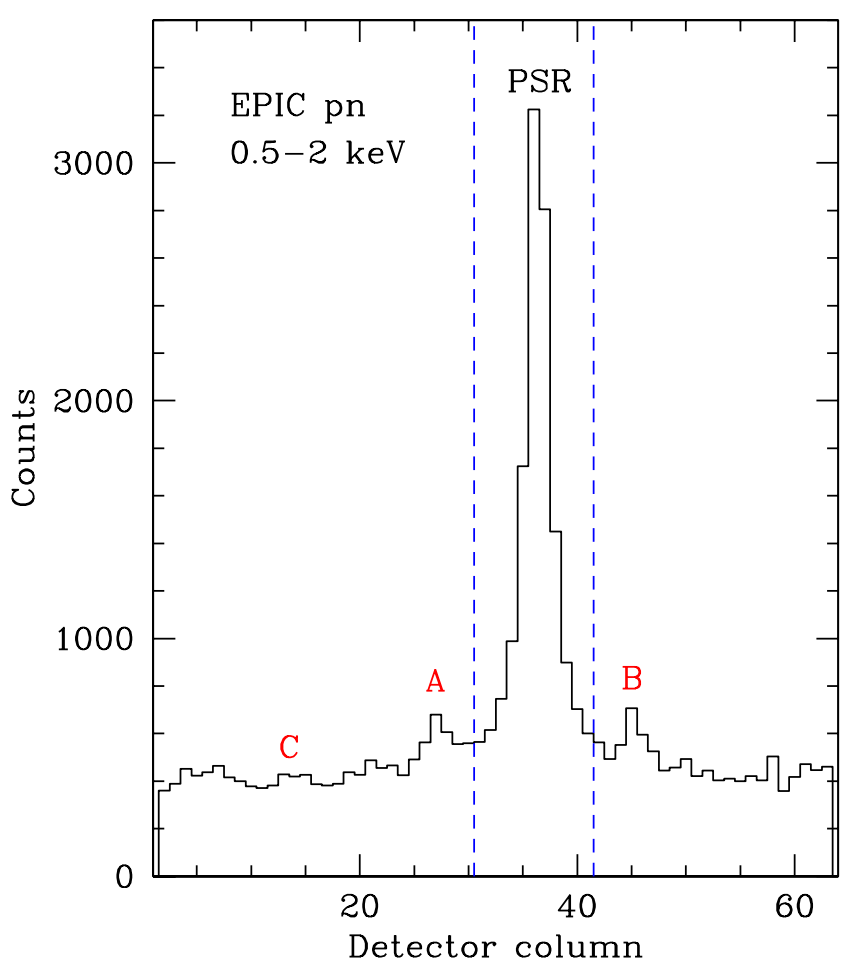

FIG. 2.- The distribution of counts from XMM-Newton EPIC pn along the imaging dimension of the fast timing mode in the $0.5-$ $2 \mathrm{keV}$ band. The pair of dashed lines mark the detector columns that the pulsar counts were extracted from. The labels show the positions of the sources from Figure 1.

pattern, pulse invariant, and flag filters were applied to produce data suitable for spectral and timing analyses.

For the spectroscopic analysis, source counts in the MOS 1 and 2 data were extracted from circles of radius 60 " centered on the radio MSP position, which enclose $\sim 90 \%$ of the total energy at $1.5 \mathrm{keV}$. The background was taken from three source-free regions surrounding the pulsar. For the spectroscopic and timing analyses of the EPIC pn dataset, the source counts were obtained from RAWX detector columns 31-41 (inclusive), equivalent to a radius of 22.5 " in the RAWX direction, which encloses $\sim 74 \%$ of the total energy for $0.3-2 \mathrm{keV}$. This relatively narrow region was chosen in order to avoid contamination from sources $\mathrm{A}$ and $\mathrm{B}$ as well as to minimize the large background level, which dominates beyond 20" from the source position, arising due to the 1-dimensional imaging mode used for the pn detector. To ensure a reliable estimate, the background was taken from three source-free regions.

\section{IMAGING ANALYSIS}

Figure 1 shows the $0.3-2 \mathrm{keV}$ coadded image from EPIC MOS1 and MOS2 of the field in the vicinity of PSR J0030+0451, as well as the relative orientation and readout direction of the EPIC pn detector. It is clear that the pulsar is the brightest source in the image. Due to the favorable telescope roll angle during the observation, the EPIC pn detector columns containing the bulk of counts from the pulsar are effectively free of contamination from other sources (Fig. 2). Sources A and B combined contribute with only a few counts $(\lesssim 0.1 \%$ of the total) in the source extraction colums of the pulsar. 
In the MOS1 and 2 images, the source count distribution around the radio position of the pulsar is fully consistent with that of a point source. Thus, we find no indication of any diffuse extended emission that could arise due to a bow shock, as seen in PSR J2124-3358 (Hui \& Becker 2006). This is not surprising given that for the combination of spin-down luminosity $(\dot{E} \approx 3 \times$ $10^{33} \mathrm{ergs} \mathrm{s}^{-1}$ ) and remarkably low transverse space velocity $\left(8-17 \mathrm{~km} \mathrm{~s}^{-1}\right.$, see Lommen et al. 2006) for this pulsar, no detectable diffuse X-ray emission is expected (see, e.g., Fig. 1 in Chatteriee \& Cordes 2002).

\section{SPECTROSCOPIC ANALYSIS}

To facilitate the spectral fitting of the MOS1 and MOS2 data, the extracted counts in the $0.1-10 \mathrm{keV}$ band were grouped so as to ensure at least 30 counts per bin. For the pn data the counts in the $0.3-3 \mathrm{keV}$ were grouped with at least 150 counts per bin. Due to the overwhealming background level, no useful spectral information is present above $3 \mathrm{keV}$ in the pn data. In addition, photons in the $0.42-0.5 \mathrm{keV}$ range were not used in order to eliminate an instrumental noise artifact specific to the fast timing mode of the pn detector.

As found in previous studies of PSR J0030+0451 (Becker \& Aschenbach 2002; Bogdanov et al. 2008; Zavlin 2007), the continuum X-ray emission from this pulsar cannot be adequately described by a single component emission model. Consequently, in the spectral fits we apply the following plausible multi-component models: i) two thermal, ii) a single thermal plus non-thermal, iii) two Comptonized thermal iv) two thermal plus one non-thermal, and v) three thermal components. For the thermal components we consider both a blackbody model and the unmagnetized $(B=0)$ $\mathrm{H}$ atmosphere model first presented in McClintock et al. (2004) and included as a private model in XSPEC. The assumption of $B=0$ is appropriate since for typical MSP magnetic fields $\left(\sim 10^{8-9} \mathrm{G}\right)$ the magnetic field does not affect the radiative opacities and equation of state of the atmosphere (see Romani 1987; Zavlin et al. 1996; McClintock et al. 2004, for details).

It has been found that blackbody fits to MSP spectra result in effective emission areas that are an order of magnitude smaller than the expected polar cap area, $R_{p c}=(2 \pi R / c P)^{1 / 2} R$, while for a $\mathrm{H}$ atmosphere they are found to be comparable (Becker \& Aschenbach 2002; Zavlin et al. 2002; Bogdanov et al. 2006a; Zavlin 2006). The discrepancy could, in principle, arise due to nonuniform heating of the polar caps. More plausably, it suggests that the surface of the polar caps is covered by a light-element atmosphere. As the spectrum of a $\mathrm{H}$ atmosphere model is harder than that of a blackbody for the same effective temperature, a blackbody model fitted to a $\mathrm{H}$ atmosphere continuum would yield a higher inferred temperature and, as a result, a smaller effective area. Nonetheless, for the sake of completeness and easier comparison with previous X-ray studies of MSPs, we have considered blackbody models in our spectral fits as well.

Zavlin et al. (2002), Zavlin (2006), and Bogdanov et al. (2007) have noted that due to the energy-dependent limb darkening effect for the atmosphere model, even for phase-integrated spectra it is

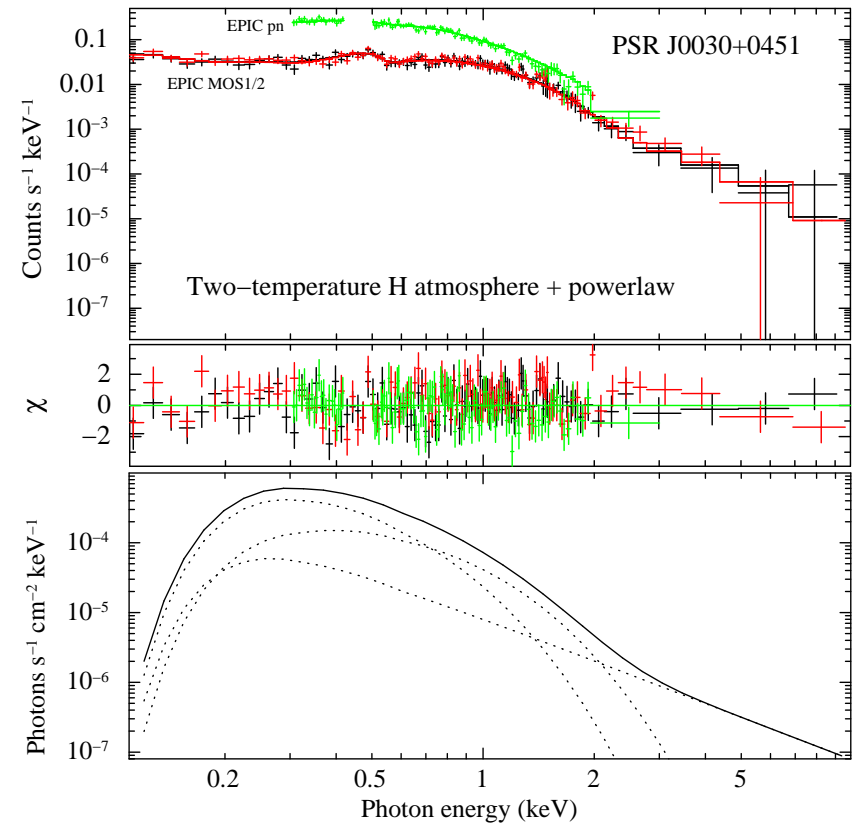

FIG. 3.- The XMM-Newton EPIC X-ray spectra of PSR J0030+0451 fitted with a two-temperature H-atmosphere plus powerlaw model. The middle panel shows the fit residuals while the bottom panel shows the best fit model. See Table 1 for best fit parameters.

necessary to take into account the rotation of the star as well as the relative orientation of the spin axis, hot spots, and the line of sight to the observer in order to ensure a reliable measurement of the temperature and emission radius (see, in particular, Fig. 2 of Bogdanov et al. 2007). For this reason, we employ the spectral model from Bogdanov et al. (2007). This model allows us to specify the angles between the spin and magnetic axes $(\alpha)$ and the spin axis and the line of sight $(\zeta)$ as well as the mass and radius of the pulsar in the spectral fits. Thus, the normalization of the thermal component(s) yields the true (deprojected and redshift-corrected) effective area of the emission region(s). In addition, the quoted best-fit temperatures represent redshift-corrected values, i.e. as they would be measured at the NS surface. For the calculation of all effective radii, we consider the parallax distance $D=300 \mathrm{pc}$. In the case of the $\mathrm{H}$ atmosphere model, we assume a neutron star with $M=1.4 \mathrm{M}_{\odot}$ and $R=12 \mathrm{~km}$. Based on the X-ray pulse profile morphology (discussed in $\S 5$ ) we also fix $\alpha=70^{\circ}$ and $\zeta=80^{\circ}$ since this combination of angles can reproduce the X-ray pulse profile shape for $M=1.4$ $\mathrm{M}_{\odot}$ and $R=12 \mathrm{~km}$. All uncertainties quoted below are given at a $1 \sigma$ level for one interesting parameter. The various spectral models and their best fit parameters are summarized in Table 1.

\subsection{Two-Temperature Thermal Spectrum}

First, we consider the two-temperature thermal model that was found to provide a good fit to the archival XMM-Newton data of PSR J0030+0451 (Becker \& Aschenbach 2002; Bogdanov et al. 2008). This model describes the new spectrum well up to $\sim 3$ $\mathrm{keV}$ but cannot fully account for the excess flux at higher energies. Thus, it is necessary to invoke alternative emission models. 
TABLE 1

Best fit SPECTRAL models and unABSORBed fluXes For PSR J0030+0451.

\begin{tabular}{|c|c|c|c|c|c|c|c|c|c|}
\hline Model $^{\mathrm{a}}$ & 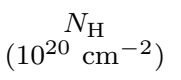 & $\begin{array}{c}T_{\text {eff, }, 1} \\
\left(10^{6} \mathrm{~K}\right)\end{array}$ & $\begin{array}{c}R_{\text {eff }, 1} \mathrm{~b} \\
(\mathrm{~km})\end{array}$ & $\begin{array}{c}T_{\text {eff, }, 2} \\
\left(10^{6} \mathrm{~K}\right)\end{array}$ & $\begin{array}{c}R_{\mathrm{eff}, 2}^{\mathrm{b}} \\
(\mathrm{km})\end{array}$ & & & $\begin{array}{c}F_{\mathrm{X}^{\mathrm{c}}} \\
(0.1-10 \mathrm{keV})\end{array}$ & $\chi_{\nu}^{2} /$ dof \\
\hline $\mathrm{BB}(\times 2)$ & $0.90_{-0.3}^{+0.3}$ & $3.05_{-0.16}^{+0.15}$ & $0.05_{-0.03}^{+0.03}$ & $1.45_{-0.08}^{+0.07}$ & $0.28_{-0.10}^{+0.13}$ & - & - & $3.1 \pm 0.1$ & $1.21 / 299$ \\
\hline \multirow[t]{2}{*}{$\operatorname{Hatm}(\times 2)$} & $2.0_{-0.3}^{+0.4}$ & $1.71_{-0.15}^{+0.16}$ & $0.25_{-0.03}^{+0.04}$ & $0.68_{-0.07}^{+0.07}$ & $2.2_{-0.6}^{+0.7}$ & - & - & $3.7 \pm 0.1$ & $1.16 / 299$ \\
\hline & & \multicolumn{8}{|c|}{-0.00} \\
\hline $\mathrm{BB}+\mathrm{PL}$ & $4.0_{-0.4}^{+0.4}$ & $2.11_{-0.04}^{+0.05}$ & $0.11_{-0.04}^{+0.04}$ & - & - & $3.09_{-0.08}^{+0.08}$ & - & $8.6 \pm 0.1(7.1 \pm 0.1)$ & $1.12 / 299$ \\
\hline $\mathrm{Hatm}+\mathrm{PL}$ & $3.4_{-0.4}^{+0.4}$ & $1.06_{-0.03}^{+0.03}$ & $0.86_{-0.30}^{+0.37}$ & - & - & $3.06_{-0.09}^{+0.09}$ & - & $6.9 \pm 0.1(4.8 \pm 0.1)$ & $1.11 / 299$ \\
\hline \multirow[t]{2}{*}{$\mathrm{BB}(\times 2)+\mathrm{PL}$} & $3.8_{-0.7}^{+0.5}$ & $2.34_{-0.36}^{+0.86}$ & $0.07_{-0.04}^{+0.05}$ & $1.75_{-0.24}^{+0.33}$ & $0.11_{-0.13}^{+0.18}$ & $3.08_{-0.09}^{+0.09}$ & - & $8.2 \pm 0.2(6.6 \pm 0.2)$ & $1.10 / 297$ \\
\hline & $1.5_{-0.3}^{+0.4}$ & $2.51_{-0.11}^{+0.11}$ & $0.08_{-0.02}^{+0.02}$ & $1.18_{-0.08}^{+0.08}$ & $0.34_{-0.17}^{+0.20}$ & 1.4 & - & $3.7 \pm 0.2(0.5 \pm 0.1)$ & $1.13 / 298$ \\
\hline \multirow[t]{3}{*}{$\operatorname{Hatm}(\times 2)+\mathrm{PL}$} & $1.9_{-0.6}^{+0.3}$ & $1.42_{-0.11}^{+0.11}$ & $0.37_{-0.01}^{+0.02}$ & $0.69_{-0.08}^{+0.08}$ & $1.9_{-0.16}^{+0.19}$ & $2.0_{-0.2}^{+0.2}$ & - & $3.9 \pm 0.4(0.6 \pm 0.1)$ & $1.12 / 297$ \\
\hline & $1.8_{-0.5}^{+0.0}$ & $1.51_{-0.07}^{+0.08}$ & $0.3_{-0.2}^{+0.2}$ & $0.70_{-0.07}^{+0.08}$ & $1.97_{-0.38}^{+0.41}$ & 1.4 & - & $3.7 \pm 0.3(0.4 \pm 0.1)$ & $1.11 / 298$ \\
\hline & & & & & & $\begin{array}{c}k T_{e} \\
(\mathrm{keV})\end{array}$ & $\bar{\tau}$ & & \\
\hline \multirow[t]{2}{*}{ Compbb $(\times 2)$} & $1.8_{-0.4}^{+0.5}$ & $2.28_{-0.10}^{+0.10}$ & $0.11_{-0.05}^{+0.06}$ & $1.03_{-0.09}^{+0.12}$ & $0.38_{-0.19}^{+0.27}$ & 100 & $0.12 \pm 0.01$ & $3.8 \pm 0.3$ & $1.10 / 298$ \\
\hline & & & & & & $\begin{array}{c}T_{\text {eff }, 3} \\
\left(10^{6} \mathrm{~K}\right)\end{array}$ & $\begin{array}{l}R_{\text {eff }, 3} \\
(\mathrm{~km})\end{array}$ & & \\
\hline $\mathrm{BB}(\times 3)$ & $1.9_{-0.5}^{+0.6}$ & $6.98_{-1.21}^{+1.40}$ & $0.004_{-0.003}^{+0.005}$ & $2.27_{-0.14}^{+0.13}$ & $0.12_{-0.06}^{+0.07}$ & $1.01_{-0.14}^{+0.12}$ & $0.46_{-0.29}^{+0.48}$ & $3.7 \pm 0.3$ & $1.10 / 297$ \\
\hline $\operatorname{Hatm}(\times 3)$ & $2.2_{-0.6}^{+0.6}$ & $3.36_{-0.78}^{+0.84}$ & $0.03_{-0.03}^{+0.06}$ & $1.05_{-0.09}^{+0.07}$ & $0.95_{-0.8}^{+0.8}$ & $0.40_{-0.07}^{+0.06}$ & $4.3_{-2.1}^{+2.8}$ & $4.0 \pm 0.4$ & $1.11 / 297$ \\
\hline
\end{tabular}

a PL is a powerlaw, BB a blackbody, and Hatm a $\mathrm{H}$ atmosphere model. All uncertainties quoted are $1 \sigma$.

b $R_{\text {eff }}$ calculated assuming a distance of 300 pc. For the $\mathrm{H}$ atmosphere model, the numbers quoted represent deprojected and redshift-corrected effective radii of one polar cap of a neutron star with $M=1.4 \mathrm{M}_{\odot}, R=12 \mathrm{~km}, \alpha=70^{\circ}$, and $\zeta=80^{\circ}$ (see text for details).

c Unabsorbed X-ray flux $(0.1-10 \mathrm{keV})$ in units of $10^{-13} \mathrm{ergs}^{-2} \mathrm{~s}^{-1}$. The value in parentheses represents the flux contribution of the powerlaw component.

\subsection{Thermal Plus Non-thermal Spectrum}

Next, we apply a composite thermal plus non-thermal (powerlaw) model. As evident from Table 1, for either a blackbody or $\mathrm{H}$ atmosphere thermal component the model yields statistically acceptable fits. However, the derived values of $N_{\mathrm{H}}$ are significantly larger than those inferred from independent measurements. In particular, the total $\mathrm{H}$ column density through the Galaxy along the line of sight to the pulsar is only $N_{\mathrm{H}} \approx 3 \times 10^{20}$ $\mathrm{cm}^{-2}$ (Dicke \& Lockman 1990). On these grounds, we deem the validity of the single temparature thermal plus non-thermal model doubtful.

\subsection{Comptonized Thermal Spectrum}

Bogdanov et al. (2006b) have proposed that the hard tail seen in the X-ray spectrum of PSR J0437-4715 may be produced by inverse Compton scattering (ICS) of the soft thermal X-rays by relativistic $e^{ \pm}$of low optical depth $(\tau \ll 1)$. Such particles are likely present in the pulsar magnetosphere above the polar caps. In the spectral fits of PSR J0030+0451, we employ the compbb Comptonized blackbody model (Nishimura et al. 1986), which includes two additional parameters: a scattering particle temperature $k T_{e}$ and optical depth $\tau$. The model assumes a plane-parallel, semi-infinite scattering medium with the source of thermal photons at the bottom. The formalism employed is valid between $k T \lesssim k T_{e} \leq 150$ $\mathrm{keV}$. For the two thermal components we link the $\tau$ parameter as both would likely be scattered by the same population of particles. For a choice of $k T_{e}=100$ $\mathrm{keV}$, the best fit parameters are $N_{\mathrm{H}}=1.8_{-0.4}^{+0.4} \times 10^{20}$ $\mathrm{cm}^{-2}, T_{\text {eff }, 1}=\left(2.28_{-0.10}^{+0.10}\right) \times 10^{6} \mathrm{~K}, R_{\text {eff }, 1}=0.11_{-0.05}^{+0.06}$ $\mathrm{km}, T_{\text {eff }, 2}=\left(1.03_{-0.09}^{+0.12}\right) \times 10^{6} \mathrm{~K}, R_{2}=0.38_{-0.19}^{+0.27} \mathrm{~km}$, $\tau=0.12 \pm 0.01$ with $\chi_{\nu}^{2}=1.10$ for 298 degrees of free- dom. The implied unabsorbed flux is $3.8 \times 10^{-13}$ ergs $\mathrm{cm}^{-2} \mathrm{~s}^{-1}(0.1-10 \mathrm{keV})$. As is generally the case for ICS, $k T_{e}$ and $\tau$ are strongly correlated so acceptible fits were obtained for a wide range of these parameters. Since we expect $k T \ll k T_{e}$, the choice of $k T_{e}$ as well as the actual energy distribution of the scattering particles do not significantly affect the X-ray spectral fits. Thus, the ICS model appears to provide a good description of the spectrum of PSR J0030+0451 as well.

\subsection{Two-Temperature Thermal Plus Powerlaw Spectrum}

Zavlin et al. (2002) and Zavlin (2006) have found the continuum X-ray emission from PSR J0437-4715 to be well described by a two-temperature thermal and a single powerlaw components. As expected based on the similar spin properties of the two pulsars, the same model provides a good description of the X-ray spectrum of PSR J0030+0451. Formally, an F-test indicates a $99.9997 \%$ probability that the addition of the powerlaw component to the two-temperature thermal model is required. As with the single temperature composite model, the derived best fit $\mathrm{H}$ column density for the two blackbody plus powerlaw model with $\Gamma$ unconstrained are in excess of the upper limit $N_{\mathrm{H}}=3 \times 10^{20} \mathrm{~cm}^{-2}$ and yield a rather steep spectral photon index $(\Gamma \sim 3)$. On the other hand, the two-temperature $\mathrm{H}$ atmosphere plus powerlaw model (Fig. 3) results in a more plausible value of $N_{\mathrm{H}}$ and a value of $\Gamma$ very similar to that found for PSR J0437$4715(\Gamma \approx 2)$. We note that due to the poor photon statistics above $\sim 4 \mathrm{keV}$, statistically acceptable fits are obtained for a wide range of photon indices (1.0-3.5 at $3 \sigma$ confidence) although for $\Gamma \gtrsim 2.5$ the implied $N_{H}$ also exceeds $3 \times 10^{20} \mathrm{~cm}^{-2}$ even for the two-temperature $\mathrm{H}$ atmosphere plus powerlaw model. 


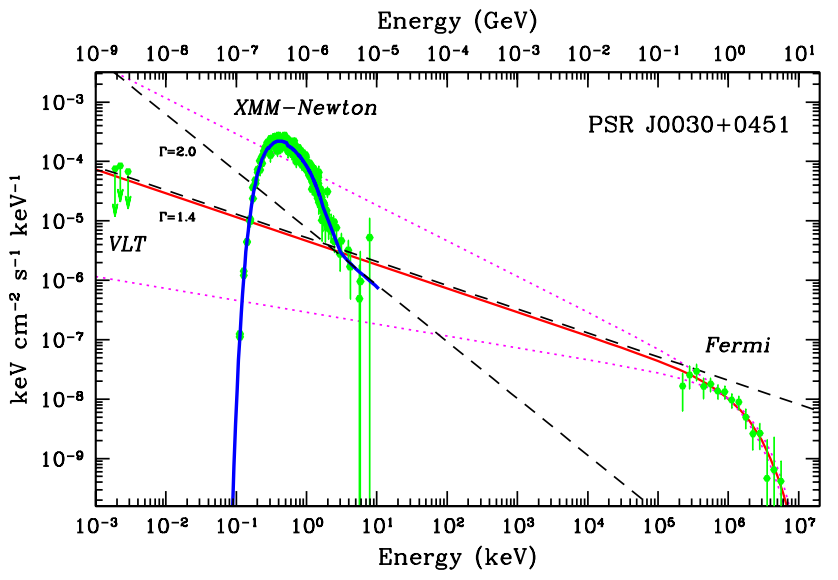

FIG. 4.- Optical-to- $\gamma$-ray spectrum of PSR J0030+0451 showing the XMM-Newton X-ray data presented in this paper, VLT optical upper limits (Koptsevich et al. 2003), and Fermi $\gamma$-ray measurements (Abdo et al. 2009) (green circles). The solid blue line shows the total absorbed two-temperature $\mathrm{H}$ atmosphere plus powerlaw model spectrum for $\Gamma=2.0$, while the red lines shows the best fit powerlaw model (solid) and uncertainties (dotted) for the Fermi LAT data. The dashed lines show the extrapolation of the best fit powerlaw spectrum with $\Gamma=2.0$ and $\Gamma=1.4$ of the twotemperature $\mathrm{H}$-atmosphere plus powerlaw model to the optical and $\gamma$-ray ranges.

\subsection{Three-Temperature Thermal Spectrum}

The high-energy portion $(\gtrsim 2 \mathrm{keV})$ of the PSR J0030+0451 X-ray spectrum could be due to an additional (third) thermal component. Indeed, for both three-temperature blackbody and $\mathrm{H}$ atmosphere models we obtain statistically acceptable fits. The best fit parameters for this model suggest the presence of a very hot and very small ( $T_{\text {eff }}=7 \mathrm{MK}$ and $R_{\text {eff }}=4 \mathrm{~m}$ for a blackbody or $T_{\text {eff }}=3.4 \mathrm{MK}$ and $R_{\text {eff }}=30 \mathrm{~m}$ for a $\mathrm{H}$ atmosphere) emission region on the neutron star surface. In principle, such a region could arise due to highly non-uniform magnetospheric heating across the polar cap surface.

\subsection{Optical-to-gamma-ray Spectrum}

Koptsevich et al. (2003) have conducted deep VLT FORS2 BVR imaging of the field surrounding the radio timing position of PSR J0030+0451. No plausible optical counterpart was detected down to $\mathrm{B} \gtrsim 27.3, \mathrm{~V}$ $\gtrsim 27.0$ and $\mathrm{R} \gtrsim 27.0$ in the immediate vicinity of the radio pulsar position. In $\gamma$-rays, Abdo et al. (2009) have reported on the Fermi LAT detection of pulsations from PSR J0030+0451 above $100 \mathrm{MeV}$. The $\gamma$-ray spectrum $(100 \mathrm{MeV}$ to $10 \mathrm{GeV}$ ) is well described by a powerlaw with a high-energy exponential cutoff with $\Gamma=1.4 \pm 0.2$ and a cutoff energy of $E_{c}=1.7 \pm 0.4 \mathrm{GeV}$.

It is interesting to examine how the X-ray spectrum extrapolates to optical and $\gamma$-ray energies for the different spectral models discussed above. Figure 4 shows the optical-to- $\gamma$-ray spectrum of PSR J0030+0451 based on the available XMM-Newton, VLT, and Fermi observations. The thermal components seen in the soft $\mathrm{X}$ ray band contribute neglegibly to the optical and $\gamma$-ray fluxes. On the other hand, for the best fit two-component $\mathrm{H}$ atmosphere thermal plus power-law model, the spectral photon index of $\Gamma=2$ grossly overestimates the optical flux (by a factor of $30-40$ ), violating the VLT upper limits, while it greatly underestimates the $\gamma$-ray flux (by a factor of $\sim 100$ ). This implies that such a powerlaw must break to a flatter powerlaw both in the UV range and between $10 \mathrm{keV}$ and $100 \mathrm{MeV}$.

Surprisingly, an extrapolation of the best fit $\gamma$-ray spectrum with $\Gamma=1.4$ (from Abdo et al. 2009) is a very close match to the best fit two-compenent thermal plus non-thermal X-ray spectrum with $\Gamma=1.4$ (see Table $1)$. Within the uncertainties of the powerlaw normalizations as well as the $\sim 20 \%$ effective area uncertainty of the Fermi LAT (see Abdo et al. 2009), the two are consistent with being identical. This suggests that if the same emission process is responsible for the hard X-ray tail and the $\gamma$-ray emission from the pulsar, then the powerlaw index has to be very close to $\Gamma=1.4$. However, given the rather large uncertainties in the two spectra, substantial improvement in the photon statistics in both energy ranges and detections at intermediate energies (10 $\mathrm{keV}$ to $100 \mathrm{MeV}$ ) are required to offer more conclusive statements regarding the relation between the $\gamma$-ray and any non-thermal X-ray emission.

Note that for the Comptonized thermal interpretation of the X-ray spectrum, one does not expect any contribution of the model components in the UV/optical nor in the Fermi band as the high-energy cutoff of the Comptonized tail is likely well below the low energy threshold of Fermi LAT $(\sim 100 \mathrm{MeV})$. Thus, for this model there is no restriction on the slope of the hard tail.

\section{TIMING ANALYSIS}

To investigate the rotation-induced modulations of the X-ray flux from PSR J0300+0451, the photon arrival times extracted from the EPIC pn source region shown in Figure 2 were first translated to the solar system barycenter using the SAS barycen tool. The arrival times were subsequently folded at the pulsar spin period using the TEMPO $^{7}$ pulsar timing package and the radio ephemeris of PSR J0030+0451 from Abdo et al. (2009).

As seen from previous X-ray timing studies of this pulsar (Becker et al. 2000; Becker \& Aschenbach 2002), the $\mathrm{X}$-ray pulse profile of PSR J0030+0451 is characterized by two peaks that are significantly broader than their radio counterparts (Fig. 5). The substantial improvement (factor of $\sim 4.5$ ) in photon statistics compared to previous observations reveals new information regarding the $\mathrm{X}$-ray pulse morphology. For instance, it is now apparent that the primary pulse is significantly stronger than the secondary, with a $\sim 13 \%$ larger peak flux in the $0.3-2$ keV range. For thermal polar cap emission this difference can be naturally produced by a geometric configuration in which one of the polar caps makes a closer approach to the line of sight to the observer. There is marginal evidence for a larger relative difference between the two pulses for $0.7-2 \mathrm{keV}$ compared to $0.3-0.7 \mathrm{keV}$, although statistically they are consistent with being identical.

Based on the lightcurve model discussed in $\S 6$, the peak-to-peak separation in phase between the two pulses is found to be $\sim 0.55$ as measured from the stronger to the weaker pulse. It is also evident that the two minima between the pulses are uneven, with a fractional difference in the minimum flux of $\sim 22 \%$ (for $0.3-2 \mathrm{keV}$ ). For a centered dipole (i.e. antipodal hot spots), Doppler boost-

\footnotetext{
7 Available at http://www.atnf.csiro.au/research/pulsar/tempo/
} 


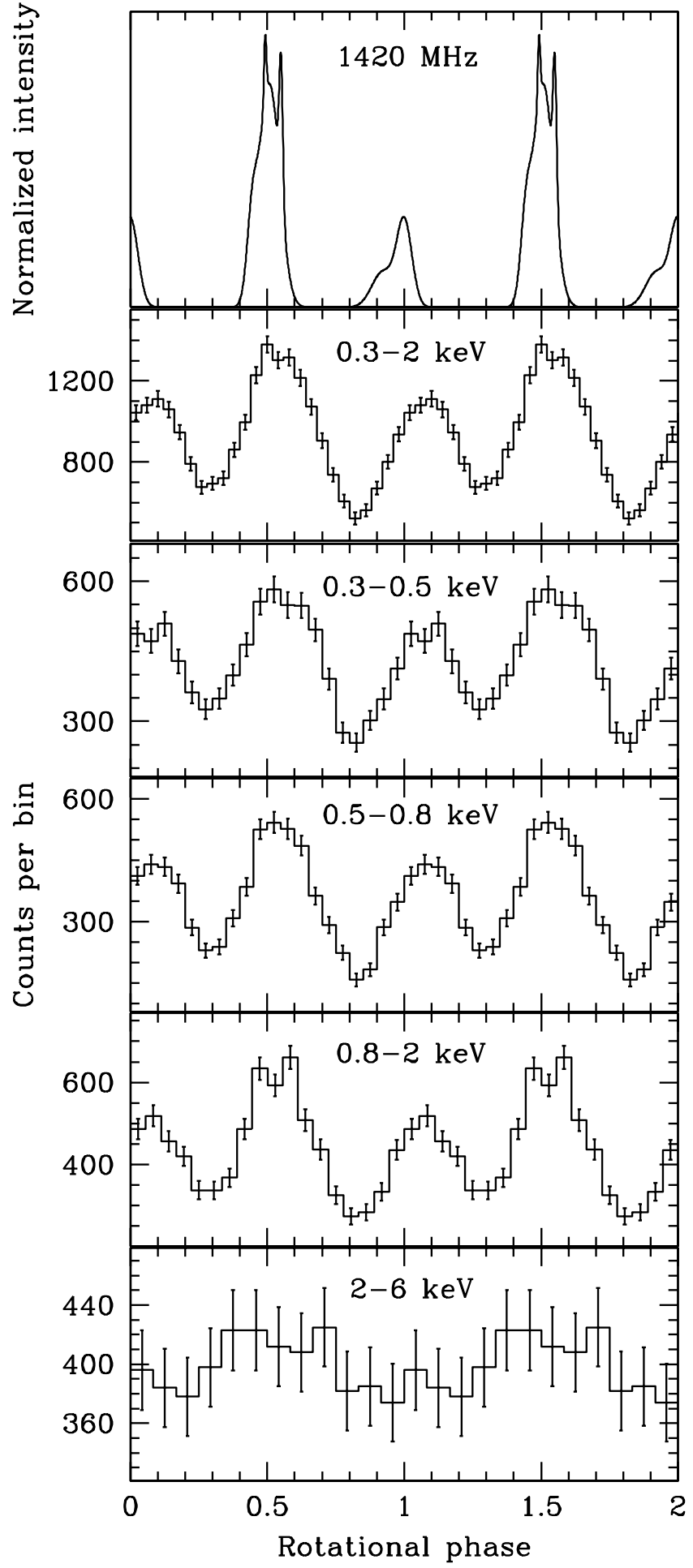

Fig. 5.- XMM-Newton EPIC pn X-ray pulse profiles of PSR J0030+0451 for different energy bands. The top panel shows the template radio pulse profile at $1420 \mathrm{MHz}$. The choice of phase zero and the alignment of the X-ray and radio profiles are arbitrary. Two rotational cycles are shown for clarity.

ing and aberration, induced by the rapid stellar rotation, can be sufficient to account for the apparent assymetry for larger stellar radii (e.g. $\gtrsim 12 \mathrm{~km}$ for $M=1.4 \mathrm{M}_{\odot}$ ). On the other hand, for more compact stars (e.g. $\lesssim 12$ $\mathrm{km}$ for $\left.M=1.4 \mathrm{M}_{\odot}\right)$ it would be necessary to invoke an off-center dipole in order to reproduce the observed asymmetry in the minima as well as the phase separation of the two pulses.

To estimate the pulsed fraction of the folded, binned, and background-subtracted lightcurves we have used two approaches: (i) from the pulse profile fits discussed in the following section and (ii) by computing the root-mean-squared pulsed fraction (see, e.g., Eq. 2 in Dhillon et al. 2009, for a definition). The former approach yields $67 \%_{-6 \%}^{+6 \%}(0.3-2 \mathrm{keV}), 71 \%_{-7 \%}^{+9 \%}(0.3-0.7$ $\mathrm{keV})$, and $63 \%_{-7 \%}^{+8 \%}(0.7-2 \mathrm{keV})$, whereas using the latter method we obtain $64 \%_{-5 \%}^{+4 \%}, 64 \%_{-5 \%}^{+6 \%}$, and $63 \%_{-6 \%}^{+6 \%}$ for the same energy bands, respectively. Although these pulsed fractions appear high, they are fully consistent with a purely thermal origin of the observed X-rays (as suggested by the spectroscopic analysis in $\S 4$ and as demonstrated in \$6) if one considers a light-element atmosphere at the neutron star surface. Note that the pulse profiles in the $0.3-0.7$ and $0.7-2 \mathrm{keV}$ energy bands are consistent with having the same pulsed fraction.

Even though the photon statistics are quite limited above $2 \mathrm{keV}$ and the profile is consistent with a constant flux, the weak modulations in the $2-6 \mathrm{keV}$ band (bottom panel of Fig. 5) are suggestive of pulsations similar to those seen at lower energies. Unfortunately, as a consequence of the relatively high background level of the EPIC pn data, no useful timing information is present above $\sim 3 \mathrm{keV}$, where the hard spectral tail begins to dominate the emission. Thus, we are unable to place any limits on the pulsed fraction for $3-10 \mathrm{keV}$, which could potentially offer clues regarding the true nature of the hard spectral tail.

\section{CONSTRAINTS ON THE NEUTRON STAR COMPACTNESS}

The spectrum and pulse profile of PSR J0030+0451 are indicative of a thermal origin of the observed Xrays. This is of particular importance since the surface thermal emission can potentially provide useful information regarding the neutron star compactness through a measurement of the mass-to-radius ratio $(M / R)$. For this purpose we employ the model from Bogdanov et al. (2007), which considers a relativistic rotating compact star with two identical, circular X-ray emitting hot spots, each (presumably) corresponding to one of the magnetic polar caps. The model also incorporates a non-rotating Schwarzschild metric as a description of the space-time in the vicinity of the star and includes Doppler boosting and propagation time delays. This relatively simple formalism is remarkably accurate for spin periods $P \gtrsim 3$ ms (Cadeau et al. 2007; Morsink et al. 2007). The NS surface is assumed to be covered by a non-magnetic, optically-thick $\mathrm{H}$ atmosphere.

Before applying the model to the X-ray pulse profiles, it was first convolved with the appropriate instrument response, while taking into account the encircled energy fraction and the sky and detector background. We fitted the pulse shape by considering the following parameters: the two temperatures and effective radii of each hot spot $\left(T_{1}, T_{2}, R_{1}\right.$, and $\left.R_{2}\right)$ the two angles $\alpha$ and $\zeta$, the stellar radius $R$, the offsets of the secondary hot spot from the antipodal position $(\Delta \alpha$ and $\Delta \phi)$, and the phase $\phi$ of the pulse peak. The hydrogen column density 


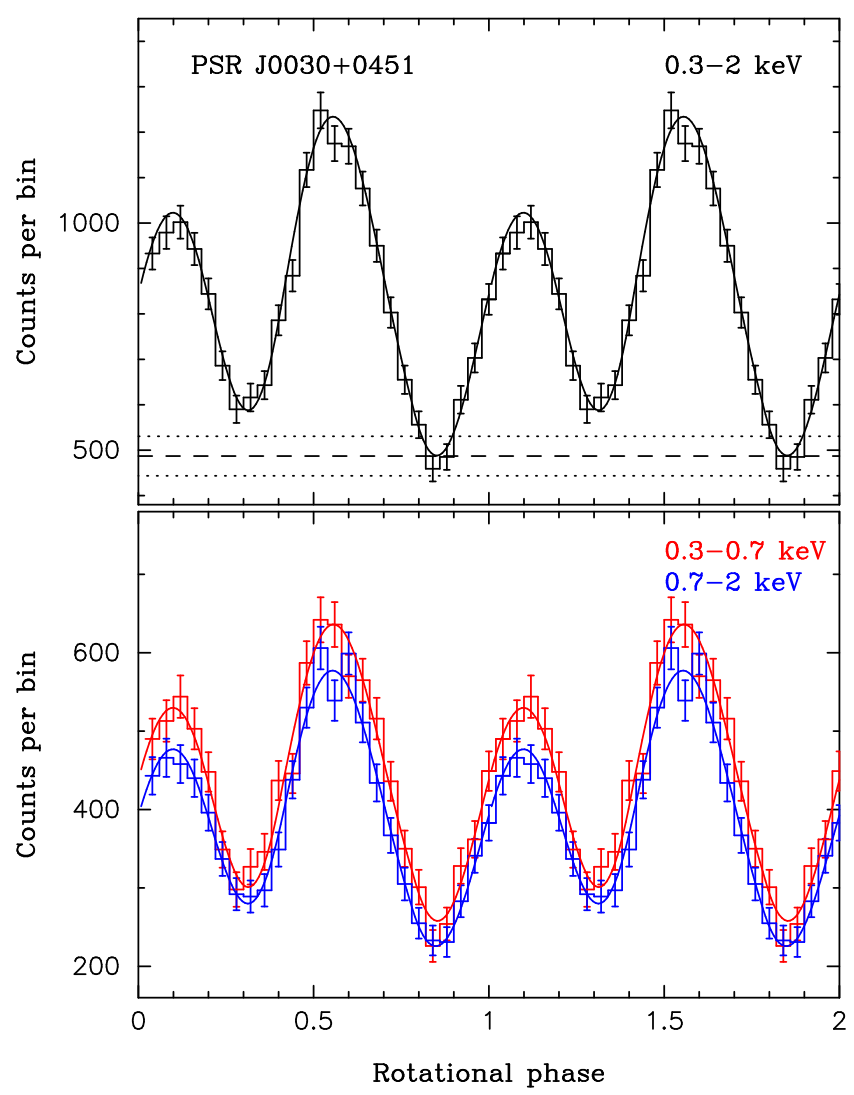

FIG. 6.- XMM-Newton EPIC pn pulse profiles of PSR J0030+0451 in the $0.3-2 \mathrm{keV}(t o p)$ and $0.3-0.7 \mathrm{keV}$ and $0.7-2 \mathrm{keV}$ (bottom) ranges fitted with a model of a rotating neutron star with two-temperature $\mathrm{H}$ atmosphere polar caps. The DC level (dashed) and uncertainties (dotted) as determined from the fits are shown in the top panel. The background level for the $0.3-2 \mathrm{keV}$ band is 314 counts per bin for 25 phase bins. See text for best fit model parameters.

along the line of sight was fixed at $N_{\mathrm{H}}=2 \times 10^{20} \mathrm{~cm}^{-2}$, while the distance was set to $D=300 \mathrm{pc}$ (Lommen et al. 2006). Varying $N_{\mathrm{H}}$ over the range $(1-3) \times 10^{20} \mathrm{~cm}^{-2}$, does not result in appreciable changes in the most interesting parameters, namely $M / R, \alpha$, and $\zeta$. The same holds true for the parallax distance uncertainty $(\sim 30 \%)$, which mostly affects the flux normalization, resulting in a larger uncertainty in the emission area. As $M / R, \alpha$, and $\zeta$ do not determine the flux normalization, the best fit values of these parameters are virtually insensitive to the distance uncertainty. Unless noted otherwise, in our analysis we assumed a fixed mass of $M=1.4 \mathrm{M}_{\odot}$ and allow $R$ to vary. The fit was performed simultaneously in two energy bands $(0.3-0.7 \mathrm{keV}$, with $0.42-0.5 \mathrm{keV}$ excluded as in the spectral fits, and $0.7-2 \mathrm{keV}$ ) in order to weaken any degeneracies between spectral parameters (temperatures and radii) from the geometric and relativistic parameters, which have no energy dependence. In order to account for any possible presence of a nonthermal component, we included the expected number of photons per bin for the best fit powerlaw spectrum into the uncertainties (assuming an unpulsed or weakly pulsed contribution). The best fit values for all free parameters were found by searching the $\chi^{2}$ hyperspace. To ensure that the obtained values correspond to the absolute minima of the parameter space, the fit was repeated

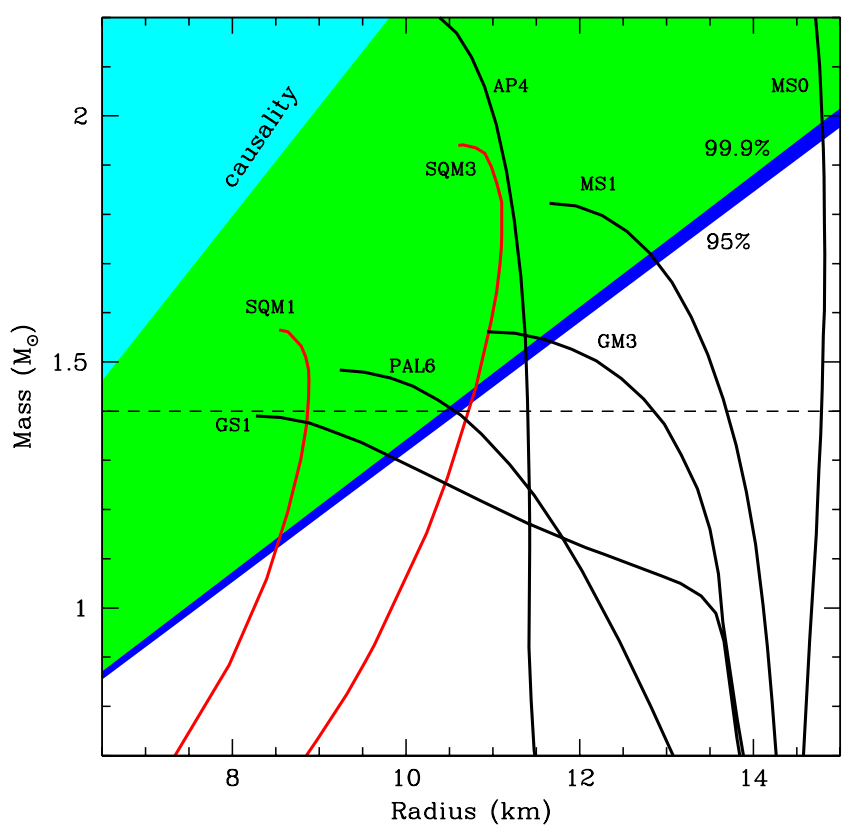

FIG. 7.- The mass-radius plane for neutron stars showing various theoretical model tracks. The regions ruled out by the X-ray data for PSR J0030+0451 at $95 \%$ and $99.9 \%$ confidence are shown in blue and green, respectively.

for 100 different combinations of initial parameter values.

The resulting best fit pulse profiles for a $\mathrm{H}$ atmosphere model are presented in Figure 6. Unlike blackbody emission, the atmosphere model is able to reproduce the pulse shape and large amplitude remarkably well. The stellar radius is constrained to be $R>10.4 \mathrm{~km}$ or, more generally, $R / R_{S}>2.5(99.9 \%$ confidence $)$ for all combinations of the other free parameters. In the case of a blackbody model, good fits to the X-ray pulse profile require implausably large stellar radii $(\gtrsim 20 \mathrm{~km})$ for a range of assumed massess $\left(M=1-2 \mathrm{M}_{\odot}\right)$. This is in agreement with the results obtained for PSR J04374715 (Bogdanov et al. 2007), PSR J2124-3358, and using the archival data for PSR J0030+0451 (Bogdanov et al. 2008).

Figure 7 shows the $M-R$ plane with a sample of predicted model track for different NS EOS from Lattimer \& Prakash (2001) and the constraints obtained for PSR J0030+0451, with the shaded regions corresponding to regions excluded by the limits on $M / R$. Although the results are consistent with all EOS if $M$ is unconstrained, it is still possible to draw some important conclusions. In particular, there is observational evidence that several recycled pulsars are more massive than the canonical $1.4 \mathrm{M} \odot$ (Freire et al. 2008a, b; Verbeist et al. 2008; Champion et al. 2008), as expected from standard MSP formation theory, which involves accretion of an appreciable amount of material. If this is true for isolated MSPs such as PSR J0030+0451 as well, then for $M \gtrsim 1.45$ the $M / R$ constraint is inconsistent with some kaon condensate EOS (e.g. GS1 for which the maximum mass consistent with our limits is $\sim 1.3 \mathrm{M}_{\odot}$ ), and most quark star EOS (e.g., SQM1-SQM3 in Fig. 7). For $M \gtrsim 1.6 \mathrm{M}_{\odot}$, the limit of $R / R_{S}>2.5$ rules out all but the stiffest equations of state (such as MS0-MS2). Conversely, if this pulsar is in fact a quark star, then 
the constraints obtained herein would require it to be $M \lesssim 1.45 \mathrm{M}_{\odot}$.

The fits to the pulse profile also provide limits on the global magnetic field and viewing geometry of the pulsar. Specifically, the pulsar obliquity is constrainted to be in the range $\alpha=38^{\circ}-90^{\circ}$, implying a substantial misalignment between the spin and magnetic axes, while the angle between the line of sight and the spin axis is $\zeta=47^{\circ}-90^{\circ}$. The relatively broad range of allowed values arises due to the strong covariance of these angles with the offset in latitude of the secondary polar cap $\Delta \alpha$, which is weakly constrained to be in the range $-42^{\circ}-52^{\circ}$. As defined, a positive value of $\Delta \alpha$ indicates a southward offset from the antipodal location. The displacement in the longitudinal direction is constrainted to be $\Delta \phi=-8^{\circ}-46^{\circ}$, consistent with both a centered and offset dipole. For this parameter, a positive value corresponds to an offset in the direction of rotation, meaning that the hot spot leads the antipodal position.

\section{CONCLUSION}

In this paper, we have presented deep XMMNewton observations of the nearby recycled pulsar PSR $\mathrm{J} 0030+0451$. For the first time we are able to fully characterize the emission spectrum of this isolated pulsar in the $0.1-10 \mathrm{keV}$ band. It exhibits striking qualitative similarities to that of PSR J0437-4715, suggesting that other typical MSPs such as the nearby J2124-3358 and J10240719 (Zavlin 2006), as well as the majority of MSPs in 47 Tuc (Bogdanov et al. 2006a), whether binary or isolated, may exhibit the same characteristic spectral shape over this energy range.

Although the spectral continuum of J0030+0451 can be adequately described by a variety of plausible models, in all instances the bulk of detected photons in the soft X-ray range $(0.1-2 \mathrm{keV})$ appear to be of thermal origin. Our $\mathrm{H}$ atmosphere polar cap model is in fair agreement with the observed continuum and pulsed emission from PSR J0030+0451. As with PSR J0437-4715 (Bogdanov et al. 2007), the relatively large pulsed fractions require the existence of a light-element atmosphere on the stellar surface and cannot be reproduced by a blackbody model for realistic NS radii. By modeling the thermal pulse shape we are able to place a limit on the allowed stellar radii of $R>10.4 \mathrm{~km}$ (99.9\% confidence) assuming $M=1.4 \mathrm{M}_{\odot}$, a substantial improvement over the constraints obtained from the shorter archival observations. As demonstrated in Bogdanov et al. (2008), even deeper X-ray observations of this and other MSPs should lead to stricter limits on $M / R$, which in turn may firmly rule out entire families of neutron star EOS. This is especially true for the growing number of binary MSPs for which complementary mass measurements from radio pulse timing are becoming available.

With future observations, it is important to uncover the true nature of the hard emission ( $\gtrsim 3 \mathrm{keV})$, through a combination of deep energy-resolved timing and phaseresolved spectroscopy at energies above $3 \mathrm{keV}$. Better characterization of the optical and $\gamma$-ray spectra of PSR J0030+0451 could potentially provide additional information regarding the origin of this emission. These endeavors have important implications for establishing MSP X-ray timing as a vible means for tight constraints on the neutron star EOS. The potential utility of MSP as precision probes of neutron star structure makes them particularly important targets for upcoming X-ray mission such as the International $X$-ray Observatory. The great increase in sensitivity of such a facility would permit detailed observations of a larger sample of MSPs than currently possible, which could ultimately lead to definitive insight into the poorly understood properties of the neutron star interior.

We would like to thank A. Lommen for providing the radio pulse profile of PSR J0030+0451 and the anonymous referee for offering numerous helpful comments. The research presented was funded in part by $X M M$ Newton Guest Observer NASA grant NNX08AD54G awarded through the Harvard College Observatory. S. B. is supported in part by a Lorne Trottier Research Chair Fellowship and a Canadian Institute for Advanced Research Junior Fellowship. This work was based on observations obtained with XMM-Newton, an ESA science mission with instruments and contributions directly funded by ESA Member States and NASA. The research in this paper has made use of the NASA Astrophysics Data System (ADS).

Facilities: XMM-Newton

\section{REFERENCES}

Abdo, A. A., et al. 2009, ApJ, 699, 1171

Becker, W. \& Aschenbach, B. 2002, Proceedings of the 270.

WE-Heraeus Seminar on Neutron Stars, Pulsars, and

Supernova Remnants, Eds. W. Becker, H. Lech, J. Trümper, p. 64

Becker, W., Trümper, J., Lommen, A. N., \& Backer, D. C. 2000, ApJ, 545, 1015

Bogdanov, S., Grindlay, J. E., Heinke, C. O., Camilo, F., Freire, P. C. C., \& Becker, W. 2006a, ApJ, 646, 1104

Bogdanov, S., Grindlay, J. E., \& Rybicki, G. B. 2006b, ApJ, 648, L55

Bogdanov, S., Rybicki, G. B., \& Grindlay, J. E. 2007, ApJ, 670, 668

Bogdanov, S., Grindlay, J. E., \& Rybicki, G. B. 2008, ApJ, 689, 407

Cadeau, C., Morsink., S. M., Leahy, D., \& Campbell, S. S. 2007, ApJ, 654, 458

Champion, D. J., et al. 2008, Science, 320, 1309

Chatterjee, S. \& Cordes, J. M. 2002, 575, 407
Dicke, J. M. \& Lockman, F. J. 1990, ARA\&A, 28, 215

Dhillon, V. S., et al. 2009, MNRAS, 394, L112

Freire, P. C. C., Ransom, S. M., Bégin, S., Stairs, I. H., Hessels, J. W. T., Frey, L. H., Camilo, F. 2008, ApJ, 675, 670

Freire, P. C. C., Wolszczan, A., van den Berg, M., Hessels, J. W. T. 2008, ApJ, 679, 1433

Harding, A. K. \& Muslimov, A. G. 2002, ApJ, 568, 862

Hui, C. Y. \& Becker, W. 2006, A\&A, 448, L13

Koptsevich, A. B., Lundqvist, P., Serafimovich, N. I., Shibanov,

Yu. A., \& Sollerman, J. 2003, A\&A, 400, 265

Lattimer, J. M. \& Prakash, M. 2001, ApJ, 550, 426

Lommen, A. N., Zepka, A., Backer, D. C., McLaughlin, M., Cordes, J. M., Arzoumanian, Z., Xilouris, K. 2000, ApJ, 545, 1007

Lommen, A. N., Kipphorn, R. A., Nice, D. J., Splaver, E. M., Stairs, I. H., \& Backer, D. C. 2006, ApJ, 642, 1012

McClintock, J. E., Narayan, R., \& Rybicki, G. B. 2004, ApJ, 615, 402 
Morsink, S. M., Leahy, D. A., Cadeau, C., Braga, J. 2007, ApJ, 663,1244

Nishimura, J., Mitsuda, K., \& Itoh, M. 1986, PASJ, 38, 819

Pavlov, G. G. \& Zavlin, V. E. 1997, ApJ, 490, L91

Romani, R. W. 1987, ApJ, 313, 718

Verbiest, J. P. W., et al. 2008, ApJ, 679, 675

Zavlin, V. E., Pavlov, G. G., \& Shibanov, Yu. A. 1996, A\&A, 315, 141
Zavlin, V. E. \& Pavlov, G. G. 1998, A\&A, 329, 583

Zavlin, V. E., Pavlov, G. G., Sanwal, D. , Manchester, R. N.,

Trümper, J., Halpern, J. P., \& Becker, W. 2002, ApJ, 56 9, 894 Zavlin, V. E. 2006, ApJ, 638, 951

Zavlin, V. E. 2007, Ap\&SS, 308, 297 